debris and blood clots are cleared and vascular blood supply is reinstated, remyelination will fail ${ }^{5}$. Thus, it is plausible that blood-borne signalling proteins, such as coagulation factors deposited at sites of physical damage, are detected by OPCs and act as surrogate markers of ongoing repair of the primary injury. This could put differentiation on hold until the damaged environment is ready for remyelination.

Demyelinated areas that arise in MS can also be considered as local 'brain injuries'. Although there is no bleeding and subsequent blood clotting involving coagulation factors in MS, chronic inflammation causes a persistent opening of the blood-brain barrier (BBB), across which these factors might pass in large amounts. Could the permanent entry of blood-borne coagulation factors prevent OPC differentiation and myelin repair?

With this question in mind, Petersen et al. revisited the observation that the soluble glycoprotein fibrinogen, which is abundant in blood plasma, is deposited in demyelinated brain regions ${ }^{2}$. First, the authors added physiological concentrations of fibrinogen to OPCs in cell culture, and showed that this coagulation factor strongly inhibited OPC differentiation and prevented axon myelination. Among the many genes in OPCs whose expression was affected by fibrinogen, the researchers detected upregulation of members of a signalling pathway known to inhibit oligodendrocyte differentiation $^{6}$ - genes encoding bone morphogenetic proteins (BMPs) and their downstream effectors, including the transcription factor ID2. Indeed, Petersen and colleagues showed that fibrinogen and ID2 could be readily visualized in regions in which remyelination had failed in the brains of people who had died with MS.

Interestingly, the authors found that OPCs exposed to fibrinogen either in vitro or in the brains of live mice often underwent a developmental switch to become a different neuronsupporting cell type called an astrocyte. This raises the possibility that astrocytic scars (a form of tissue growth that occurs in response to injury in MS brains and that might prevent myelin repair) arise from a switch in OPC identity. Such a hypothesis will require testing in mouse models of MS.

Fibrinogen drives the activation of brainspecific immune cells, which can indirectly inhibit remyelination. However, the effects reported by Petersen and co-workers are direct: they result from fibrinogen binding to the BMP type I receptor protein ACVR1 on the surface of OPCs to stimulate the BMP signalling cascade in these cells (Fig. 1). This is of interest because inhibitors of BMP signalling have already been developed. Indeed, the authors provide evidence that one such inhibitor can counteract the detrimental effects of fibrinogen on OPC differentiation, pointing to a possible avenue for therapy.

In addition, fibrinogen itself might be a drug target. Petersen et al. show that the fibrinogen-cleaving enzyme ancrod - an anticoagulant from a snake venom that has been proposed (although not approved) as a treatment for ischaemic stroke - enhanced the remyelination of demyelinated axons. A mouse model of MS has previously been shown to benefit from ancrod and fibrinogen depletion ${ }^{7}$, owing in part to anti-inflammatory effects. However, it is possible that myelin repair is also improved in these animals. Regardless of the relative contributions of indirect and direct effects of ancrod on OPCs, clinical tests would be needed to determine the drug's efficacy in people with MS. Unfortunately, given that the drug is offpatent, such trials are unlikely to find support in the pharmaceutical industry.

It is becoming apparent that coagulation factors do much more than simply act in the blood-coagulation cascade. The research group that performed the current study has previously shown ${ }^{8}$ that the enzyme thrombin, which cleaves fibrinogen to produce fibrin, is activated in demyelinated tissue. This leads to the formation of large fibrin complexes, which are equivalent to blood clots. Moreover, tissue plasminogen activator protein, which is routinely given to people who have had an ischaemic stroke to promote the breakdown of fibrin-containing blood clots, inhibits the death of oligodendrocytes ${ }^{9}$ and promotes axonal regeneration ${ }^{10}$. One must assume that these factors, like fibrinogen, access the brain in the absence of a functional BBB, and have roles in determining the success or failure of myelin repair. And although fibrinogen is apparently not expressed in the brain, other coagulation factors are ${ }^{8}$. Their uncontrolled transfer from the blood when the BBB leaks will no doubt perturb the 'coagulation-unrelated' functions of these factors in the brain; these effects await exploration.

If a compromised $\mathrm{BBB}$ is an entry port for blood-borne inhibitors of myelination, does fibrinogen entry reduce cortical myelination and affect higher brain functions in chronic conditions other than MS? The brains of people with Alzheimer's disease have a leaky $\mathrm{BBB}$ and show fibrinogen infiltration ${ }^{11}$. Individuals carrying a form of the $A P O E$ gene that increases the risk of Alzheimer's disease display reduced $\mathrm{BBB}$ integrity, and this variant has been associated with age-dependent myelin breakdown ${ }^{12}$. Petersen and colleagues' findings might thus have implications beyond MS - these should be investigated soon.

Klaus-Armin Nave is in the Department of Neurogenetics, and Hannelore Ehrenreich is in the Department of Clinical Neuroscience, Max Planck Institute of Experimental

Medicine, 37075 Göttingen, Germany.

e-mail:nave@em.mpg.de

1. Petersen, M. A et al. Neuron 96, 1003-1012 (2017)

2. Yates R. L. et al. Ann Neurol. 82, 259-270 (2017).

3. Pfeiffer, S. E., Warrington, A. E. \& Bansal, R. Trends Cell Biol. 3, 191-197 (1993)

4. Gibson, E. M., Geraghty, A. C. \& Monje, M. Dev. Neurobiol. http://dx.doi.org/10.1002/ dneu.22541 (2017)

5. Yuen, T. J. et al. Cell 158, 383-396 (2014).

6. Cole, A. E., Murray, S. S. \& Xiao, J. Stem Cells Int http://dx.doi.org/10.1155/2016/9260592 (2016).

7. Adams, R. A. et al. J. Exp. Med. 204, 571-582 (2007)

8. Davalos, D. et al. Ann. Neurol. 75, 303-308 (2014).

9. Correa, F. et al. J. Exp. Med. 208, 1229-1242 (2011)

10.Zou, T. et al. J. Neuropathol. Exp. Neurol. 65, 78-86 (2006).

11.Ryu, J. K. \& McLarnon, J. G. J. Cell. Mol. Med. 13, 2911-2925 (2009).

12. Bartzokis, G. et al. Arch. Gen. Psychiatry 63, 63-72 (2006).

This article was published online on 13 December 2017.

\title{
Nanoscale interfaces made easily
}

\begin{abstract}
Methods for making interfaces between atomically thin sheets of materials might open the way to a range of nanotechnologies. A practically simple method has been reported, based on the cyclical switching of gaseous reagents. SEE LETTER P.63
\end{abstract}

\section{WEIJIE ZHAO \& QIHUA XIONG}

A tomically thin sheets of semiconducting materials, known as two-dimensional semiconductors, have outstanding potential for making low-power, high-speed electronic and optoelectronic devices ${ }^{1-3}$, including flexible electronics. Such applications often require heterostructures: interfaces formed between two or more
2D semiconductors, which can either stack on top of each other (vertical heterostructures) or be joined at their edges (lateral heterostructures). Versatile and scalable techniques for the mass production of heterostructures are therefore required. On page 63, Sahoo et al. ${ }^{4}$ report a substantial advance that allows the controllable growth of seamless, highquality lateral heterostructures made from widely studied 2D semiconductors known as 


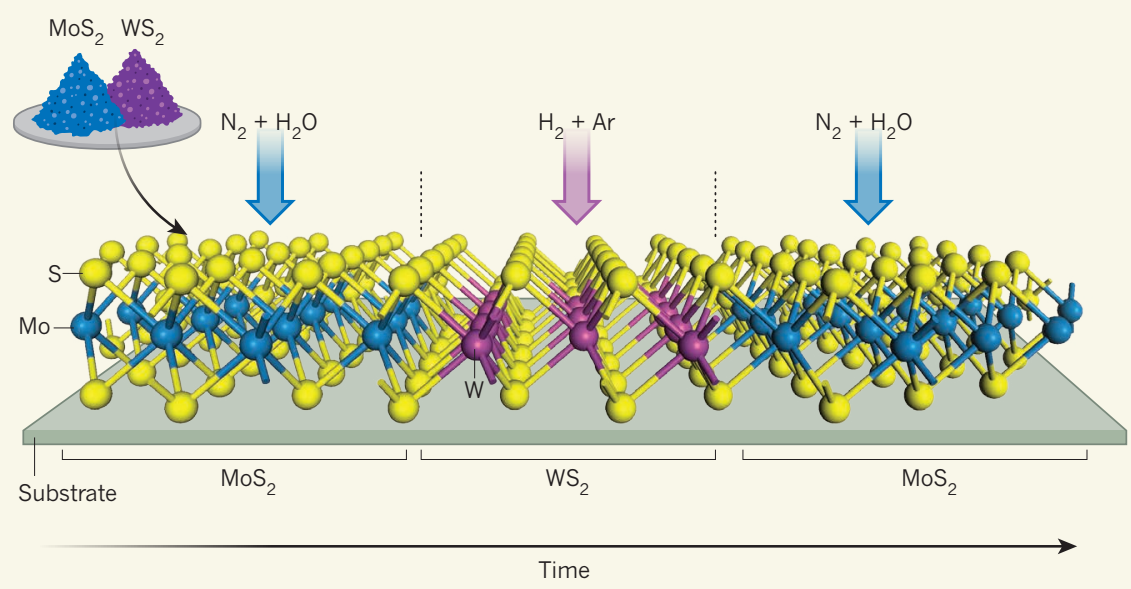

Figure 1 | A strategy for growing lateral multi-junction heterostructures. Interfaces between the edges of atomically thin sheets of different semiconductors are called lateral heterostructures, and have potential technological applications. Sahoo et al. ${ }^{4}$ report a method for making lateral heterostructures from compounds known as transition-metal dichalcogenides (TMDs), which include molybdenum disulfide $\left(\mathrm{MoS}_{2}\right)$ and tungsten disulfide $\left(\mathrm{WS}_{2}\right)$. The authors heat a mixture of two powdered TMDs in a furnace, and pass carrier gases over them (coloured arrows). The carrier gases react with the TMDs to produce gaseous intermediates (not shown), which then react on the surface of a substrate to deposit sheets of the TMDs. When a mixture of nitrogen and water vapour is used as the carrier gas, only $\mathrm{MoS}_{2}$ forms. When the carrier gas is switched to a mixture of hydrogen and argon, the growth of $\mathrm{MoS}_{2}$ is terminated and $\mathrm{WS}_{2}$ grows at the edge of the pre-grown $\mathrm{MoS}_{2}$. By switching cyclically between the carrier gases, 2D multi-junction heterostructures are produced.

transition-metal dichalcogenides (TMDs).

Transition-metal dichalcogenides have the general formula $\mathrm{MX}_{2}$, in which $\mathrm{M}$ is molybdenum $(\mathrm{Mo})$ or tungsten $(\mathrm{W})$ and $\mathrm{X}$ can be sulfur (S) or selenium (Se). Lateral TMD heterostructures can be constructed by 'stitching' the edges of two TMD sheets together using covalent bonds. In the past few years, there has been a flurry of papers ${ }^{5-9}$ reporting methods for synthesizing TMD lateral heterostructures using edge epitaxial growth, a method that allows a second TMD to grow at the edge of another, pre-grown TMD crystal. These heterostructures can be fabricated into $\mathrm{p}-\mathrm{n}$ junctions, which conduct currents in only one direction (a property known as rectification), and constitute one of the building blocks of modern electronic and optoelectronic devices. Twodimensional $\mathrm{p}-\mathrm{n}$ junctions hold great promise for the development of atomically thin devices such as light-emitting diodes, solar cells and integrated circuits (chips).

Lateral TMD heterostructures have previously been made in one-step procedures ${ }^{5,6}$ that lacked the flexibility to make multi-junction heterostructures or more than one type of heterostructure, or in two-step or multi-step processes that involve many changes of TMD precursors and reaction chambers ${ }^{7-9}$. Sahoo and colleagues' method overcomes those constraints in a 'one-pot' procedure - a process that allows several steps to be performed in one reaction chamber. One of the many advantages of their strategy is the operational simplicity with which different TMDs can be selectively grown.

The authors' approach builds on a method known as chemical-vapour deposition (CVD), in which a substrate is exposed to gaseous precursor compounds (sometimes mixed with carrier gases) that react or decompose on the substrate to deposit the targeted solid product at an optimal temperature and pressure. The researchers found that $2 \mathrm{D} \mathrm{MoX}_{2}$ and $\mathrm{WX}_{2}$ can be grown sequentially from a mixture of powders of the two compounds, thus forming lateral heterostructures, simply by switching the carrier gases in the CVD growth chamber (Fig. 1).

The secret to success lies in the intriguing and complicated chemical reactions that occur between the carrier gases and the powdered TMD solids. The reactions produce highly volatile species such as hydroxides and oxides, which undergo redox reactions at distinct rates to deposit $\mathrm{MoX}_{2}$ or $\mathrm{WX}_{2}$ selectively, depending on the carrier gases used. When the carrier is a mixture of nitrogen and water vapour, the growth of only $\mathrm{MoX}_{2}$ is promoted. But when the carrier is switched to a mixture of hydrogen and argon, the volatile molybdenum compounds are quickly depleted by reactions with the hydrogen, so that only $\mathrm{WX}_{2}$ grows. By switching carrier gases multiple times, as many alternating domains of $\mathrm{MoX}_{2}$ and $\mathrm{WX}_{2}$ as desired can be prepared - corresponding to a sequence of lateral heterostructures.

Sahoo and co-workers used high-resolution transmission electron microscopy to show that some types of junction in their heterostructures were seamless and atomically sharp. They also used spectroscopic techniques to confirm the alternating pattern of TMD domains, to verify that each domain contains just one type of TMD, and to show that the junctions in the heterostructures are made reproducibly.

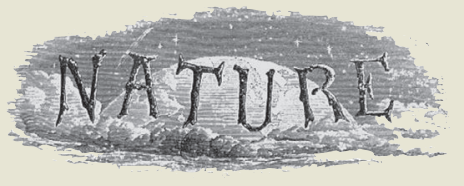

50 Years Ago

As good trade unionists know, wage claims at times of economic belt-tightening are no more successful than whistling in a blizzard. However sweet the music sounds, it never carries far. The Association of University Teachers is far from being a trade union; if it were, it would probably not have persisted with its claim that teachers in universities are underpaid. The British Government has rewarded the association for its pains by asking the Prices and Incomes Board, a notoriously unsentimental body, to undertake a survey of university salaries ... if the Prices and Incomes Board should conclude that there are no grounds for an increase, that is likely to be an end to the matter. And once the board ... have the bit between their teeth, no government is going to feel moved to set up a review body more sympathetic to the teachers. From Nature 6 January 1968

\section{Years Ago}

The Science Museum, South Kensington, was re-opened to the public on Tuesday, January 1. The museum has been closed to the public for nearly two years; it has, however, been open without interruption for students. As compared with 1914 conditions, the extent and the hours of opening for 1918 are somewhat reduced, but the greater part of the museum will be open free on every weekday from 10 a.m. to 5 p.m ... The collections contain many unique objects of great interest as representing discoveries, inventions, and appliances that have been of first-rate importance in the advancement of science and of industry. Such objects as Watt's engines, early locomotives, steamships ... and textile machinery are records of British contributions to the progress of the world. From Nature 3 January 1918 
The authors went on to demonstrate that their technique could be used to make multijunction lateral heterostructures for compounds known as TMD ternary alloys (which contain one type of metal, but a mixture of sulfur and selenium atoms). To do this, the authors used a powdered mixture of $\mathrm{MoSe}_{2}$ and $\mathrm{WS}_{2}$, or of $\mathrm{MoS}_{2}$ and $\mathrm{WSe}_{2}$ (rather than a mixture of $\mathrm{MoS}_{2}$ and $\mathrm{WS}_{2}$, or of $\mathrm{MoSe}_{2}$ and $\mathrm{WSe}_{2}$, as in their first experiments). This produced highquality, 2D lateral heterostructures consisting of domains containing the alloys $\mathrm{MoS}_{2(1-x)} \mathrm{Se}_{2 x}$ or $\mathrm{WS}_{2(1-x)} \mathrm{Se}_{2 x}$ (where $x$ is a number less than 1). The optical and electrical properties of such heterostructures could now be fine-tuned by altering the alloy composition ${ }^{10}$.

The authors conducted preliminary electrical characterizations of single-junction heterostructures produced using their method. They observed that planar $\mathrm{p}-\mathrm{n}$ junctions that formed at the boundaries of electron-doped $\mathrm{MoX}_{2}$ (made by adding a small amount of electrons to $\mathrm{MoX}_{2}$ ) and hole-doped $\mathrm{WX}_{2}$ (formed by removing a few electrons from $\mathrm{WX}_{2}$ ) show good rectification behaviour, which is a further indication of the high quality of the heterostructures. They also observed photodiode behaviour - the generation of a substantial current when the junction area was illuminated by light. Having the ability to build such tiny $\mathrm{p}-\mathrm{n}$ diodes and photodiodes holds great potential for future efforts to miniaturize electronic and optoelectronic devices.

Sahoo and co-authors' method opens up a promising route for the synthesis of highquality lateral heterostructures. Insights into the thermodynamics and chemistry operating at the atomic scale in this process are now needed to develop the ability to prepare heterostructures involving any desired combination of TMDs. Moreover, research must be performed to work out why interfaces that switch from $\mathrm{MoX}_{2}$ to $\mathrm{WX}_{2}$ are not as sharp as those in which $\mathrm{WX}_{2}$ switches to $\mathrm{MoX}_{2}$, and to optimize the production of sharper $\mathrm{MoX}_{2}-\mathrm{WX}_{2}$ interfaces.

It will also be important to explore variations of the technique that might allow the growth of lateral heterostructures between $\mathrm{MX}_{2}$ and other exotic 2D materials, including those that have metallic, semi-metallic or superconducting properties $^{1,2}$, to make new types of device. The availability of complex TMD heterostructures - including those that have several junctions in series - should also allow the exploration of fundamental physics, such as the mechanism by which charge transfer occurs at interfaces. Lastly, Sahoo and co-workers' technique will enable the development of proof-of-concept prototype devices, to advance our knowledge of the viability and scope of 2D technologies.

Weijie Zhao and Qihua Xiong are in the Division of Physics and Applied Physics, School of Physical and Mathematical Sciences, Nanyang Technological University, 637371

Singapore.

e-mail:qihua@ntu.edu.sg

1. Novoselov, K. S. et al. Proc. Natl Acad. Sci. USA 102, 10451-10453 (2005)

2. Geim, A. K. \& Grigorieva, I. V. Nature $4999,419-425$ (2013).

3. Ajayan, P., Kim, P. \& Banerjee, K. Phys. Today 69 , 38-44 (2016)

4. Sahoo, P. K., Memaran, S., Xin, Y., Balicas, L. \& Gutiérrez, H. R. Nature 553, 63-67 (2018)

5. Gong, Y. et al. Nature Mater. 13, 1135-1142 (2014).

6. Huang, C. et al. Nature Mater. 13, 1096-1101 (2014).

7. Duan, X et al. Nature Nanotechnol. 9, 1024-1030 (2014).

8. Li, M.-Y. et al. Science 349, 524-528 (2015).

9. Zhang Z. et al. Science 357, 788-592 (2017).

10.Feng, Q. et al. Adv. Mater. 26, 2648-2653 (2014).

\title{
More than one way to a central nervous system
}

\author{
Have the molecular mechanisms that are linked to the developmental \\ organization of centralized nervous systems evolved once or multiple times? \\ Evidence from nine animal species points to the latter. SEE ARTICLE P. 45
}

\section{CAROLINE B. ALBERTIN}

\section{\& CLIFTON W. RAGSDALE}

A nimal nervous systems come in many shapes and sizes, ranging from a handful of neurons to large, complex brains. A key question has been whether the centralized nervous systems found in many bilaterally symmetrical animals (bilaterians), which include vertebrates and insects, share a common evolutionary origin, or evolved more than once. At a superficial level, both flies and vertebrates boast a brain connected to a single nerve cord that extends into the trunk. In addition, molecular data indicate that key regulatory genes are deployed similarly during nervous-system development in vertebrates, flies ${ }^{1}$ and another bilaterian, a segmented worm 